\title{
A framework for the systematic realization of phenomena for enhanced sensing of radiological and nuclear materials, and radiation.
}

\author{
M J F Healy \\ Cranfield Forensic Institute, Cranfield University, Defence Academy of the United Kingdom, \\ Shrivenham, Swindon, Wiltshire, SN6 8LA. \\ E-mail: m.j.f.healy@cranfield.ac.uk
}

\begin{abstract}
The quest for new sensing phenomena continues because detecting, discriminating, identifying, measuring and monitoring nuclear materials and their radiation from greater range, at lower concentrations, and in a more timely fashion brings greater safety, security and efficiency. The potential phenomena are diverse, and those that have been realized can be found in disparate fields of science, engineering and medicine, which makes the full range difficult to realize and record. The framework presented here offers a means to systematically and comprehensively explore nuclear sensing phenomena. The approach is based on the fundamental concepts of matter and energy, where the sequence starts with the original nuclear material and its emissions, and progressively considers signatures arising from secondary effects and the emissions from associated materials and the environment. Concepts of operations such as active and passive interrogation, and networked sensing are considered. In this operational light, unpacking nuclear signatures forces a fresh look at the sensing concept. It also exposes how some phenomena that exist in established technology may be considered novel based on how they could be exploited rather than what they fundamentally are. This article selects phenomena purely to illustrate the framework and how it can be best used to foster creativity in the quest for novel phenomena rather than exhaustively listing, categorizing or comparing any practical aspect of candidate phenomena.
\end{abstract}

\section{Introduction}

Radiological and nuclear materials have widespread use in medicine, industry [1], energy [2] and defence [3]. Exploiting their benefits involves sensing applications such as exploration, mining, process monitoring and waste assay. Nuclear hazards arise through accidents [4], war [5] and terrorism [6,7] and defending against these hazardous materials involves tasks ranging from acquiring intelligence to assuring decontamination [8]. Whilst early published work on nuclear detection by non-traditional signatures [9] was arguably driven by the fear of terrorism [10], the civil nuclear energy renaissance and concerns of nuclear weapons proliferation [11,12] are increasingly important drivers for seeking enhanced nuclear sensing. Clear benefits will arise across all sectors from an enhanced capacity to sense radioactive and nuclear materials from greater range, at lower concentrations, with greater specificity, with more precise spatial attribution, in a more timely fashion and with greater operational versatility than is currently possible.

For highly radioactive materials the major hazard is likely to be the emitted radiation, but in other cases the material itself may be the hazard, which could be through chemical toxicity or a fissile property suitable for nuclear weapons. Sensing radiation is more common-place than sensing the source material, but the two are inherently linked; radiation is the usual means to locate and identify the source material but then the location and nature of the source material is used to calculate the spatial distribution and longevity of the radiation field. Techniques that increase the range from which primary radiation can be detected also tend to increase the sensitivity and timeliness for detecting the source should it be nearby. However, increased sensing range can reduce the capacity to locate the source precisely. For most current detection systems it is the transport properties of primary emissions such as alpha particles that limit the ability to detect hazards, hence researchers seek phenomena that generate strong signatures with more versatile transport properties that are more effectively detected by technology. The term versatile may refer to signatures that have greater range in air, greater penetration through container materials, that do not travel in straight lines or even that persist after the source has been removed. There are a multitude of potentially useful phenomena, but before their potential can be investigated, they must first be realized. This is not trivial as the phenomena and technologies to exploit them are diverse and arise in disconnected disciplines, thus any exploration for suitable phenomena is prone to omissions. The main purpose of this paper is to outline an approach by which phenomena for sensing radiological and nuclear material and the hazardous radiation they emit can methodically and comprehensively be brought to mind. 


\section{The basis of the framework}

The framework supporting the systematic realization of phenomena for enhanced sensing of radiological and nuclear materials and radiation appears as figure 1. For ease of communication, radiological materials will be encompassed within the term nuclear material for most of the article. The framework is based on working outward from the nuclear material, considering the interactions of the only two tangibles known; energy and matter (including anti-matter), wrapped within the operational concepts of passive sensing and active interrogation. This approach works through five categories each with two sections $(a$ and $b)$ and each section has further divisions ( $i$ and $i i$ ). Section $a$ generally refers to the property itself and section $b$ generally refers to an effect that is indicative of that property. Division $i$ refers to passive sensing and division $i i$ refers to active interrogation, which have been included to reflect important operational issues in radiation sensing. An example issue could be that an intelligence lead operation may need to be covert and thus active interrogation may warn that sensing is in progress. In the case of continuous wide area alert then active interrogation could subject many people to long radiation exposure, or require too much energy. Active interrogation is not restricted to probing the nuclear material alone, this framework encourages active interrogation to include probing any region containing relevant information in the form of matter or energy.

The main purpose of the framework is to foster imaginative thinking and support a comprehensive realization of useful phenomena. It is not claimed that the categories, sections, and divisions in this systematic approach are perfectly distinct, as there are phenomena that could be realized under multiple fields. Perhaps there are modifications to this framework that could be made to improve its utility for sorting and recording phenomena. But for the purpose of this paper, an ambiguity in where a phenomena belongs in this framework may indicate multiple opportunities to exploit the signature, corroborate information, alert to potential interferences, or just provoke deeper thought in the concept of sense. Connecting lines on the right hand side of figure 1 remind us that the signatures are usually only indicators of the nuclear material or radiation and that there may be considerable deduction needed to infer the presence, location, nature, abundance of a material or radiation. The arrows show the direction information flows in order to deduce nuclear material and radiation from signatures. The deduction process should consider the potential false positives and false negatives arising from the fundamental signature and from the nature of the deduction. The prospect of false indicators, the prospect of viable sensing technology, and the prospect for robust implementation in the field environment are factors which must be considered when choosing a novel phenomenon to select for development and exploitation. 


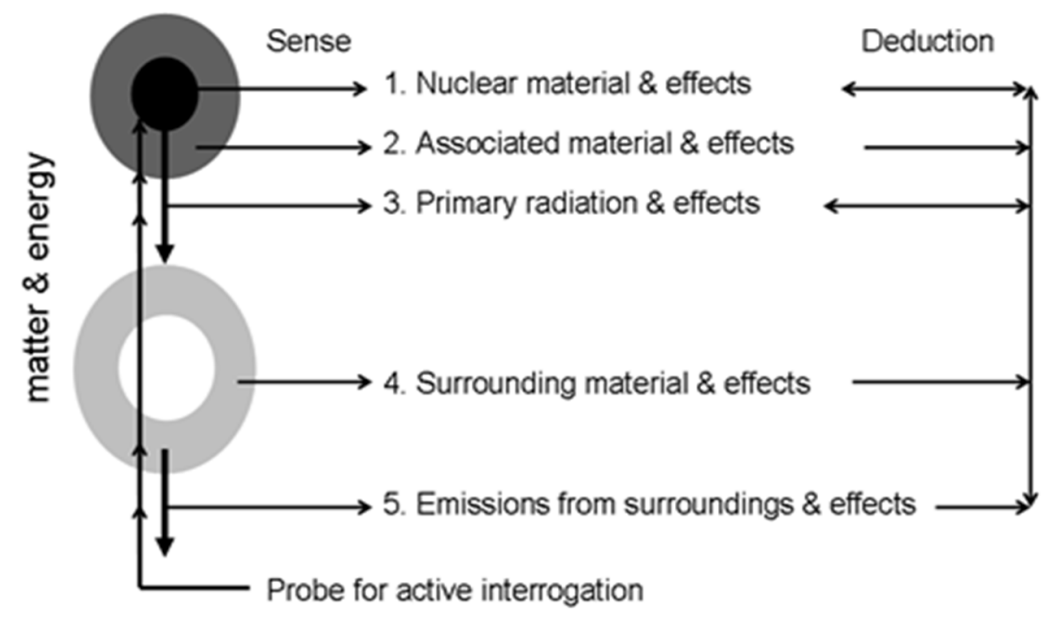

\section{Description of Category 1-5, Section a/b, Division i/ii}

1. The original nuclear material

a. i. Passive sampling of the nuclear material

ii. Active sampling of the nuclear material

b. i. Passively observed effects of the nuclear material

ii. Active interrogation of effects of the nuclear material

2. Material associated with the nuclear material, inc. catalysis \& fission etc.

a. i. Passive sampling of associated material

ii. Active sampling of associated material

b i. Passively observed effects of the associated material

ii. Active interrogation of effects of associated material

3. Primary radiation from the nuclear material

a. i. Radiation passively emitted from original nuclear material

ii. Primary radiation itself actively probed

OR (Radiation from nuclear material, induced by probe)

b. i. Passively observed effects of primary radiation

ii. Active interrogation of effects of primary radiation

4. Surrounding material affected by nuclear material and primary radiation

a. i. Passive sampling of affected material

ii. Active sampling of affected material

b. i. Passively observed effects of affected material

ii. Active interrogation of effects of affected material

5. Emissions from surrounding material

a. i. Passively emitted radiation from surrounding material

ii. Emissions from surrounding material induced by external probe

b. i. Passively observed effects of emissions from surrounding material

ii. Active interrogation of effects of emissions from surrounding material

\section{Illustrative example}

Co-located mass spectrometry

MS on material ablated by a remote laser

Deflection of cosmic muons

Deflection of muons from a muon probe

Airborne 'canary' products monitored from fixed sites.

Sampling of ${ }^{133} \mathrm{Xe}$ by directed aircraft

Health effects of $\mathrm{Pb},{ }^{6} \mathrm{Li}$, or Be on humans

Radiography of lead concealment

Alpha, beta, gamma, neutron, anti-neutrinos, heat Spatial interference / temporal beats

Neutron Activation Analysis

Witnessing radiation sickness arising in humans

Extracting DNA for damage analysis

Chemical sampling of generated ozone

Use of calixarenes to scavenge free radicals

Agglomeration of charged particles, ie 'cloud chamber'

Opacity arising in optical fibre assessed with laser

Radiation induced air fluorescence

Optically or thermally stimulated luminescence

Fluorescence arising from prior fluorescence

Figure 1.

A framework supporting the systematic realization of phenomenon for enhanced sensing of radiological and nuclear materials and their radiation hazards. 


\section{Illustrating the framework with selected phenomena}

\section{Category 1. The original nuclear material}

Category 1 refers to properties and effects of the original nuclear material. Section $a$ refers to characteristic properties of the nuclear material such as density, atomic mass, nuclear structure, electronic structure etc. and there are many techniques to determine these. Fundamentally, section $a$ aims to foster imaginative sampling strategies to co-locate the nuclear material and the sensor of the sensing system. The sampling strategy is considered passive (division $i$ ) if the sample and sensor become co-located without any intervention. An example could be the application of mass spectrometry to waterborne effluent downstream from a nuclear facility, where material unaccounted for (MUF) may be indicative of the grade of nuclear fuel manufactured upstream. In this case, although material arrives passively at the sensor, the flow of a river or the direction of wind could be used to infer the geographical origin of the material. Examples of active interrogation (division ii) would include removal of a sample by inspectors, or sending a sensor directly into the nuclear environment, or some situation where a laser could be used to ablate a sample at distance to liberate material then analyzed downwind. Some characterization techniques relevant to this section are nuclear reaction analysis [13] which identifies material through properties of its nuclear structure, and mass spectrometry [14,15] which identifies material by its atomic mass.

Section $b$ refers to an effect arising from the nuclear material, an illustrative phenomenon being the chemical toxicity of depleted uranium [16]. Another phenomenon is electrostatic force between very heavy nuclei and muons, where severe muon deflection would indicate the presence of very high $\mathrm{Z}$ material, quite possibly fissile. This phenomenon has been put forward as a means to screen cargo for special nuclear materials [17]. Should the muons originate naturally, as cosmic muons do, then the phenomenon could be considered passive and hence realized under division $i$. However division $i i$ is concerned with active interrogation, and so stepping through this framework raises the question whether muons could be produced artificially. A high flux directional muon source would allow much simpler detector arrangements and algorithms to be used, and nuclear materials to be detected and appraised with greater speed and sensitivity.

\section{Category 2. Material associated with the nuclear material}

Category 2 refers to materials that are associated with nuclear materials. The association may be weak and only indicate a nuclear sensing possibility that needs further confirmation by traditional means. An example is the element lead which is often used for shielding, where a large lead vessel with a cavity may suggest a strong source is concealed within. Examples of materials that have associations with special nuclear materials (SNM) are ${ }^{6} \mathrm{Li}$ and Be. Other examples may be fission products such as ${ }^{85} \mathrm{Kr}$, ${ }^{129} \mathrm{I},{ }^{131} \mathrm{I}$ and ${ }^{133} \mathrm{Xe}$ (sometimes called canary products [18]). An associated material more characteristic of the original nuclear material may be the residual material after a long series of radioactive decays. The specific ratio of daughter products can potentially indicate the time and place the source was processed.

Section $a$ represents sampling the associated material itself, and there are many traditional materials characterization techniques for this. Passive sampling (division $i$ ) may be atmospheric sampling routinely conducted at ground based establishments supporting the comprehensive test ban treaty (CTBT) [19]. Whereas airplanes dispatched to sample downwind of events may be considered active interrogation under division $i$.

Section $b$ represents effects arising from the associated material. An illustration of an effect that is passively observed (hence division $i$ ) is the adverse effect that lead and beryllium have on human health. An illustrative example of a division $i$ i phenomenon would be the strong attenuating effect that lead has on X-rays where the lead is actively probed by an X-ray beam in radiography [20].

\section{Category 3. The primary radiation from the nuclear material}

Category 3 encompasses the traditional means to sense nuclear materials via the primary emissions such as alpha, beta, gamma, and neutrons and their effects [21,22]. However, there are more exotic forms of emissions such as anti-neutrinos [23,24] that may be exploited as technology evolves to detect them. Technologies may develop from research in astronomy or high energy physics. Also, there are less exotic emissions such as heat that can already be effectively sensed, but the abundance of thermal signatures from non-nuclear objects can give an impractical rate of false positives. Maybe heat is best applied to finding sources in open countryside, or used in conjunction with imaging techniques as happen with nuclear fuel containers [25]. Category 3 phenomena support the bulk of current sensing capability, and so should be considered as the capability benchmark for novel phenomena. However, alpha, beta, gamma and neutron radiations still have great unrealized potential for enhanced sense through miniaturization, cost reduction and enhanced communication. Abundant widespread covert 'energy harvesting' networked traditional sensors, some of which may be close to the source [26], could negate the need for long range sensors in many applications. Traditional category 3 phenomena are likely to be needed to confirm the presence of a nuclear material found by novel means, and there have been recent improvements in gamma detector sensitivity and discrimination through improved sensor materials [27] and artificial spectral sharpening algorithms [28]. Also, although people often state a desire to find the nuclear material, actually it is the radiation that is usually the health hazard and so category 3 addresses the actual property most deleterious 
to health rather than the sometimes irrelevant material origin of the hazard.

Section $a$ refers to primary radiation itself and division $i$ refers to it arising passively such as from natural radioactive decay. Also, emissions that arise without intent could be considered as passive; an example being neutron, gamma or antineutrino emissions from the routine operation of a nuclear reactor. Division $i i$ refers to primary radiation arising from active interrogation. An illustrative phenomenon is the fission process of SNM induced by a probing beam of neutrons or photons, where gamma spectroscopy is used to detect this process and infer the presence of the fissile material [29]. Active interrogation can enhance the sensitivity with which SNM can be detected at fixed distances, or it can be used to extend range in applications such as maritime interdiction. Under active interrogation we should include the possibility of interrogating the emitted primary radiation itself rather than just inducing it. Light is characterized using light as a probe in Michelson interferometry, and sound waves are used to probe sound via the phenomenon of beats, so sensing radiation by actively probing it has precedence [30].

Section $b$ refers to effects of primary radiation. Sadly it should be remembered that most radiological accidents are only discovered following the reporting of symptoms such as nausea following human exposure. The many passively observable effects of acute radiation sickness (ARS) [31] are illustrative of division $i$ phenomena. There are recent advances in the sensitivity and speed that genetic damage can be observed through gene expression analysis [32] which requires DNA to be extracted from the body. This targeted extraction could be considered active interrogation under division $i$.

\section{Category 4. The surrounding material affected by the nuclear material and its radiations.}

Nuclear material may affect its surroundings directly via mechanisms such as chemical catalysis, or indirectly via primary radiation which may liberate electrons and form free radicals or long polymer chains.

Section $a$ refers to the sampling of modified surrounding material. A passive technique (ie division $i$ ) may be the use of static chemical sensors to detect and monitor ozone created by the action of radiation on oxygen. Interrogative techniques (division ii) may include sampling modified material by actively scavenging it and concentrating it, perhaps using calixarenes which are a subgroup of molecules that selectively trap ions or smaller molecules [33]. Airborne material may or may not travel further than conventional alpha, beta and gamma radiation, but airborne material has the capacity to turn corners and escape unsealed cargo containers. Thus scavenging airborne material helps sensors to function where there is no direct line-of-sight from source to sensor.

Section $b$ refers to effects of the surrounding material. The cloud chamber [34] is an example of apparatus where surrounding gas particles are ionized by primary radiation (passively, hence division $i$ ). These act as condensation centres seeding visible droplets. An example of actively interrogating an effect of surrounding material (hence division ii) exists in the field of fibre optics. The transparency of glass is degraded by exposure to radiation and the extent of this effect can be interrogated with a laser. Lasers can not only be used to quantify damage but they can also show how far along a fibre the damage has occurred. Long distance communication fibres are widespread and spatially well defined, so probing the opacity of a national optical fibre network with laser light could enable intense radiation fields to be identified and located on a national scale. However, fibre degradation could occur for reasons other than radiation, many false positives are possible.

\section{Category 5. Emissions from surrounding material}

This category bears some similarity to category 3 in that it refers to wave or particle emissions, but from the surrounding material rather than the nuclear material itself. An illustrative example of a surrounding material that may be excited is nitrogen in air. Section $a$ refers to emissions from the surrounding material whilst section $b$ refers to effects arising from these emissions.

An illustrative emission (i.e. section a) that is passive (i.e division i) would be ultraviolet photons released after nitrogen atoms have been excited by radiation. This process of fluorescence can be imaged at great distance by optical systems tuned to the right wavelength with narrow band-pass filters $[35,36]$. Section $a$ division $i i$ refers to emissions that are actively induced by external probes, or where the emission itself is actively interrogated (similar to category 3 section $a$ division ii). An induced emission could be light emitted when radiation damaged materials are annealed. This technique of thermo-luminescence is already widely used in dosimetry [37], but in dosimetry the materials are considered components of the detection system. These materials do not have to be part of the monitoring system, they could be gemstones in jewellery or ceramics in mobile phones, or bespoke materials placed strategically in the environment. Thermo-luminescence is usually considered a short range technique because of its relative lack of sensitivity, however the retained memory of radiation exposure can be used to extend range. For instance, with good distribution and collection strategies then abundant small simple thermo-luminescent sensors can achieve widespread distribution and effective penetration into the environment. Alternatively, simple thermo-luminescent materials placed in a cargo container may accompany a potential nuclear material in close proximity for a long time [38] and hence the accumulated signature may become strong enough to generate an alert. The detection opportunity of traditional sensors only at ports may be too brief to do this. These are examples of how one attribute (in this case a memory effect) could be transformed into other attributes (range and sensitivity) by imaginative processes.

Section $b$ refers to effects of emissions from surrounding material (the principle is similar to that of category 3 section $b$.) Emissions from the surrounding material are likely to be less energetic and abundant than the primary radiation and so their effects are likely to be weaker too. However, the emissions may have greater penetration than the primary radiation (as often occurs with 
fluorescence signatures generated below the absorption edge) so even weak effects may be observed at distance. Strong secondary effects cannot be completely excluded as they do exist, an example being the strong secondary X-ray radiation created by absorption of primary laser radiation in a hohlraum (radiation cavity) used in inertial confinement fusion. Similarly the hot air and debris of a nuclear explosion fireball arise from a long series of absorptions and re-radiations of energy in surrounding material. A passively observed effect of the emissions from surrounding material (division $i$ ) is the temporary screening of the thermal pulse from a nuclear weapon (which gives some idea of the weapon's construction). With considerable imagination, the fact that a nuclear fireball obscures radiowaves is a phenomena that could be considered active interrogation (hence division ii). This extreme example is used simply to illustrate a division of this framework, and is perhaps so complex and tenuous that it indicates the framework should cascade no further from the original nuclear material.

\section{Discussion}

This framework helps in the search for enhanced sensing capability, including completely new phenomena and new ways to exploit known phenomena. It is likely that other ways to deconstruct the nuclear and radiation sensing problem exist. There may be methods that group phenomena more distinctly, use terms less subjective than 'effect', and include operational factors beyond passive and active interrogation. Perhaps a framework could be developed that does not anchor itself to a notion of 'layers' away from the original material. This 'layering' is a spatial representation of mass and energy that actually is not distributed this way. The approach chosen for this framework was devised to help realize novel phenomena, where subdividing the problem concentrates the mind into unfamiliar channels to promote imagination and to ensure some spread of endeavor across the problem. For that purpose the overlap between the sections is no drawback, but instead offers multiple opportunities with multiple perspectives to prompt ideas. Perhaps the existence of category 3 (dedicated to traditional radiation) is unnecessary, and photon radiation could be considered as an effect under $1 \mathrm{~b}$ and particle radiation could be considered an associated material under category 2 . However, damaging ionizing radiation is so important in itself that it warrants consideration in its own right, and the technology and processes to detect it are so mature that not exploiting the body of knowledge bespoke to it would be wasteful. Although category 3 'ring fences' traditional radiation as a concept, the four subdivisions within it and the two subsequent categories 4 and 5 force a fresh look at traditional radiation. Generally, section b (effects) of all categories is in some ways already accommodated in subsequent categories, perhaps the clearest example being that category 4 could simply be included in section $3 \mathrm{~b}$.

A key route to identifying novel phenomena is challenging what people consider to be the sensor. For instance, the phenomena of fluorescence is not considered novel when alpha particles liberate photons from zinc sulphide inside a traditional detector. Whereas the fluorescence phenomenon is considered novel when the fluorescing material (air in some cases) is instead distant from the operator, close to the radiation source, and only the optical sensor is near the operator. In many instances, phenomenon already involved in traditional detection (everything from the agglomeration in a cloud chamber to thermoluminescence) could be reconsidered novel when the sensing material is present in the environment rather than inside the instrument and observed remotely. Perhaps examining conventional laboratory analysis instruments, and considering how to maximize the spatial separation of components from each other and the nuclear material would suggest ways that known phenomena could be exploited for long range detection. This discussion frequently highlights 'range' as an attribute that we wish to enhance, but the framework is general, and the enhancement of many other properties may be far more important, such as selectivity in the case of forensics.

Importantly, many of the novel signatures the author has considered can arise from sources other than nuclear materials or radiation. Detection techniques that rely on novel phenomena are likely to give high rates of false positives and are likely to be vulnerable to being intentionally exposed to signatures that cause some mode of failure. It is possible that highly characteristic novel phenomenon will become exploitable, but until then, perhaps the best route to exploit novel phenomena will be through orthogonal techniques that compensate for each other's weaknesses. For the foreseeable future, traditional radiation sensing will have a strong role confirming the indicators generated by novel phenomenon. The lines and arrows in figure 1 which illustrates the framework, highlights that considerable deduction may be required to transform the signals from phenomena into reliable information on the existence, abundance, spatial distribution, etc. of nuclear materials and radiation

\section{Conclusion}

This paper has presented a framework to help realize and explore phenomena that could offer enhanced sensing of radiological and nuclear materials and their radiation. The five categories of the proposed systematic approach cascade outward from the nuclear material, where each category contains two sections which are matter/energy and the effects of matter/energy. Each section contains two divisions representing the operational modes of passive sensing and active interrogation. The categories, sections and divisions have been populated with phenomena chosen to illustrate the nature of the framework and to show how it can help stretch the imagination in sensing concepts. It has been shown that the framework fails if an attempt is made to use it to uniquely categorize phenomena, but (although not shown here) no phenomena realized by an international working group were found to be incompatible with this system. Pursuing this systematic approach with the aid of figure 1 has helped identify new phenomena for 
enhanced sensing of nuclear material and radiation. The framework has also invoked creative thinking on how phenomena that have been employed in traditional ways can be utilized differently, to enhance sensing in terms of range, accessibility, sensitivity, versatility, persistence, selectivity and spatial attribution. Breaking the problem of seeking novel phenomena into these twenty components allows us to exhaustively explore a concept before moving our attention onward. The framework offers an 'uncomfortable lens' to help force us to look at the familiar problem of nuclear sensing in a fresh way. The article also offers a warning that novel signatures may not always generate the information originally sought; they may be prone to accidental or intentional false positives, and this must be factored in at the outset of choosing phenomena to exploit.

\section{Acknowledgements}

The author thanks Lt Col Mauricio Moutinho Silva of the CBRN Defence Division, Brazilian Army Technological Centre for his valuable discussions, his perspective and his appreciation for detail. The author also wishes to thank NATO and the UK Department of Energy and Climate Change for funding travel to share this concept with working groups at NATO and the IAEA.

\section{References}

[1] International Atomic Energy Agency, "Categorization of Radioactive Sources," IAEA Safety Standards Series No. RS-G-1.9. Vienna, Austria, July. 2005. STI/PUB/1227. ISBN 92-0-103905-0

[2] M.M. Abu-Khader, "Recent advances in nuclear power: A review," Progress in Nuclear Energy, vol. 51, pp. 225-235, 2009.

[3] B. Tertrias. "Defense of Deterrence: The Relevance, Morality and Cost-Effectiveness of Nuclear Weapons," IFRI NonProliferation Papers, Paris, France, 2011.

[4] J.-C. Nenot, "Radiation accidents over the last 60 years," Journal of Radiological Protection, vol. 29, pp. 301-320, 2009.

[5] S. Glasstone and P.J. Dolan, "The Effects of Nuclear Weapons," Government Printing Office. Washington, D.C., 1977.

[6] M. Bunn, Y. Morozov, R. Mowatt-Larssen, S. Saradzhyan, W. Tobey, V.I. Yesin, P.S Zolotarev, "Report for Belfer Center for Science and International Affairs," Harvard Kennedy School, Institute for U.S. and Canadian Studies, Jun. 2011.

[7] P.A. Karam, "Radiological Terrorism," Human and Ecological Risk Assessment, vol. 11, pp. 501-523, 2005.

[8] M.J.F. Healy, K. Weston and M. Romilly, “A Model to Support CBRN Defence," Defense \& Security Analysis, vol. 25, no. 2, pp. 119-135, Jun. 2009.

[9] C.E. Moss, R.M. Goeller, D.F. Milligan, J.E. Valencia and J. Zinn, "Remote sensing of radiation," Nuclear Instruments and Methods in Physics Research A, vol. 422, pp. 832-836, 1999.

[10] C.W. Hughes, "Japan's Aum Shinrikyo, the changing nature of terrorism, and the post-cold war security agenda," Pacifica Review: Peace, Security \& Global Change, vol. 10, no. 1, pp. 39-60, 1998.

[11] S. Varrall, “The Next Nuclear Wave: Renaissance or Proliferation Risk?” Global Change, Peace \& Security, vol. 24, no. 1, pp. 127-140, 2012.

[12] Working paper submitted by the United Kingdom of Great Britain and Northern Ireland to the 2005 Review Conference of the Parties to the Treaty on the Non-Proliferation of Nuclear Weapons, "Verification of nuclear disarmament: final report on studies into the verification of nuclear warheads and their components," NPT/CONF.2005/WP.1, Apr. 18, 2005

[13] J.R. Tesmer and M. Nastasi (eds.), "Handbook of Modern Ion Beam Materials Analysis". Materials Research Society, Pittsburgh, PA, pp. 139-204, 1995.

[14] J.S. Becker, H.-J. Dietze, "State-of-the-art in inorganic Mass Spectrometry for Analysis of High-Purity Materials," International Journal of Mass Spectrometry, vol. 228, pp. 127-150, 2003.

[15] J.F. Wacker, N.A. Wogman, K.B. Olsen, S.L. Petersen, O.T. Farmer, J.M. Kelley, G.C. Eiden and T.C. Maiti, "Ultra Trace Analysis of Uranium and Plutonium by Mass Spectrometry," Presented at the International Conference on Advances in Destructive and Non-Destructive Analysis for Environmental Monitoring and Nuclear Forensics, Karlsruhe, Germany, Oct 21-23, 2002, Paper IAEA-CN-98/3/02.

[16]E.S. Craft, A.W. Abu-Qare, M.M. Flaherty, M.C. Garofolo, H.L. Rincavage, M.B. Abou-Donia, "Depleted and Natural Uranium: Chemistry and Toxicological Effects," Journal of Toxicology and Environmental Health, Part B, vol. 7, pp. 297317,2004

[17] S. Pesente, S.Vanini, M. Benettoni, G. Bonomi, P. Calvini, P. Checchia, E. Conti, F. Gonella, G. Nebbia, S. Squarcia, G. Viesti, A. Zenoni and G. Zumerle, "First Results on Material Identification and Imaging with a Large-Volume Muon Tomography Prototype," Nuclear Instruments and Methods in Physics Research A, vol. 604, pp. 738-746, 2009.

[18] J.B. Johnson, S.W. Reevea, W.A. Burnsa and S.D. Allena, "Optical Detection of Special Nuclear Materials: An Alternative Approach for Standoff and Remote Sensing," in Proc. of SPIE, vol. 7665, 76651L-6, 2010.

[19] H.S. Miley, S.M. Bowyer, C.W. Hubbard, A. D. McKinnon, R.W. Perkins, R.C. Thompson and R.A. Warner, "Automated Aerosol Sampling and Analysis for the Comprehensive Test Ban Treaty," IEEE Transactions on Nuclear Science, vol. 45, no. 3, Jun. 1998

[20] G. Chen, G. Bennett and D. Perticone, "Dual-energy X-ray Radiography for Automatic High-Z Material Detection,” Nuclear Instruments and Methods in Physics Research B, vol. 261, pp. 356-359, 2007.

[21] G.F. Knoll, "Radiation Detection and Measurement," John Wiley \& Sons, 2010, ISBN-13: 978-0470131480 
[22] R. Klann, J. Shergur and G. Matteisch, "Current State of Commercial Radiation Detection Equipment for Homeland Security Applications," Nuclear Technology, vol. 168, pp. 79-88, Oct. 2009.

[23] B. Cabrera-Palmer, D. Reyna, L. Sadler, J. Lund, S. Kiff, N.S. Bowden, A. Bernstein and S. Dazeley, "Advances Towards Readily Deployable Antineutrino Detectors for Reactor Monitoring and Safeguards," Presented at the First International Conference on Advancements in Nuclear Instrumentation Measurement Methods and their Applications, Marseille, France, Jun. 7-10, 2009.

[24] N.S. Bowden, A. Bernstein, M. Allen, J.S. Brennan, M. Cunningham, J.K. Estrada, C.M.R. Greaves and C.D. Winant, "Experimental Results from an Antineutrino Detector for Cooperative Monitoring of Nuclear Reactors," Nuclear Instruments and Methods in Physics Research A, vol. 572, no. 2, pp. 985-998, 2007.

[25]F.J. Madruga, D.A. Gonzalez, J.M. Mirapeix and J.M.L. Higuera, “Application of Infrared Thermography to the Fabrication Process of Nuclear Fuel Containers," NDT\&E International, vol. 38, pp. 397-401, 2005.

[26] D. Stephens, Jr. and A. Peurrung, "Detection of Moving Radioactive Sources Using Sensor Networks," IEEE Transactions on Nuclear Science, vol. 51, no. 5, pp 2273-2277, 2004.

[27] P. Guss, M. Reed, D. Yuan, A. Reed and S. Mukhopadhyay., "CeBr3 as a Room-temperature, High-resolution Gamma-ray Detector," Nuclear Instruments and Methods in Physics Research A, vol. 608, pp. 297-304, 2009.

[28] L.J. Meng and D. Ramsden, "An Inter-comparison of Three Spectral-Deconvolution Algorithms for Gamma-ray Spectroscopy," IEEE Transactions on Nuclear Science, vol. 47, no. 4, pp. 1329-1336, 2000.

[29] M. Owen, G. Weston and J. O'Malley, "AWE Development of Active Interrogation Techniques for the Detection of SNM," in Proc. of SPIE, vol. 7304, 73041K-1, 2009

[30] S. Nagai, “Acoustic Power Measurement an Optical Heterodyne Method,” Ultrasonics, vol. 23, no. 2, pp.77-82, 1985.

[31] K.L. Koenig, R.E. Goans, R.J. Hatchett, F.A. Mettler, Jr, T.A. Schumacher, E.K. Noji and D.G. Jarrett, "Medical Treatment of Radiological Casualties: Current Concepts," Annals of Emergency Medicine, vol. 45, no. 6, pp. 643-652, 2005.

[32] M.A. Chaudhry, "Biomarkers for Human Radiation Exposure," Journal of Biomedical Sciences, vol. 15, pp. 557-563, 2008.

[33] Y.K. Agrawal, J.P. Pancholi and J.M. Vyas, ”Design and synthesis of calixarenes" Journal of Scientific \& Industrial Research Vol. 68, September 2009, pp. 745-768

[34] R.P. Shutt, "Cloud Chambers: Expansion and Diffusion," Nuclear Instruments and Methods, vol. 162, pp. 379-388, 1979.

[35] S.M. Baschenko, "Remote Optical Detection of Alpha Particle Sources," Journal of Radiological Protection, vol. 24, pp. 7582, 2004.

[36] E. Inrig, L. Erhardt, V. Koslowsky, B. Andrews, H. Ing, M. Dick and P. Forget, “An Air Fluorescence Imaging System for the Detection of Radiological Contamination," in Proc. of SPIE, vol. 8018, 80180G-1, 2011.

[37] V. Kortov, "Materials for Thermoluminescent Dosimetry: Current Status and Future Trends," Radiation Measurements, vol. 42, pp. 576-581, 2007.

[38] G. Janssens-Maenhout, F. De Roob and W. Janssens, "Contributing to Shipping Container Security: Can Passive Sensors Bring a Solution?” Journal of Environmental Radioactivity, vol. 101, pp. 95-105, 2010. 